3 Research Square

\title{
Attitude of Medical Students Towards Neurology, Faculty of Medicine, University of Khartoum 2019: A Cross-Sectional Study.
}

\author{
Abdelhaleem Mohammed \\ University of Khartoum \\ Muneer Musa ( $\nabla$ muneerabzootk96@gmail.com) \\ University of Khartoum
}

\section{Research Article}

Keywords: Neurophobia, Neurology specialty, Neurology teaching

Posted Date: January 22nd, 2021

DOl: https://doi.org/10.21203/rs.3.rs-136183/v1

License: (c) (1) This work is licensed under a Creative Commons Attribution 4.0 International License.

Read Full License 


\section{Abstract}

Background: Medical students have unfavorable attitude towards Neurology and often perceive it as the most difficult medical specialty. This perception is described as " neurophobia " in the medical literature and its effects and potential solutions are being investigated in many countries around the world.

Methods: A cross sectional study was conducted in which a questionnaire was administered to a sample of third, fourth, fifth and sixth years' medical students at Faculty of Medicine, University of Khartoum. The sample size was 310 selected through stratified, systematic random sampling technique. In this study, the questionnaire includes the following aspects: demographic data, assessment of the level of interest in Neurology as a future career, evaluating the satisfaction with the neurosciences course, identifying the factors which affect the attitude of students towards Neurology, highlighting the most useful strategies in teaching Neurology.

Results: Females constituted $71 \%$ of the respondents. $37.4 \%$ of the students had little or no interest and only $9.4 \%$ were strongly interested in Neurology. $46.8 \%$ of the students graded their satisfaction with the course as neutral (i.e.: moderate) and $30.6 \%$ were unsatisfied with the delivered course $(6.8 \%$ of them were strongly unsatisfied). Limited exposure to neurological patients and separation of basic sciences teaching from clinical application were considered as the top contributing factors to formation of this negative perception towards Neurology. Increased exposure to neurological patients, three dimensional simulators, video recordings, online resources as well as improving teaching skills of the lecturers were labeled as the most fruitful strategies to make progress in Neurology teaching.

Conclusion: This study reveals that 'neurophobia' probably affects a considerable proportion of medical students in Khartoum University. It reflects the possible contributing factors; Besides It encourages the stakeholders to modify the current approach to neuroscience and neurology education, and in this regard, it puts forward several strategies to make an evolution in this arena.

\section{Background}

Neurology is the branch of medicine concerned with the study of structure, function and disorders of the nervous system. Practically this knowledge is applied in diagnosis and management of these diseases. Medical students often perceive neurology as the most complicated medical specialty. This perception is renowned as "neurophobia" in the medical literature. The term neurophobia was first invented by Jozefowicz in 1994 to characterize the fear of neurosciences and clinical neurology that is attributed to the students' incapability to apply their knowledge of basic sciences to clinical practice (1). While Jozefowicz did not provide scientific data to support his claim, evidence for neurophobia has now been concluded from different sources. Several multinational studies have investigated students' attitude toward neurology teaching and practice focusing on risk factors and possible solutions. Reviewing the results of these studies, neurophobia manifests to be a global phenomenon that affect students in various stages of medical educations $(2)(3)(4)(5)(6)(7)$. The previous studies have cited poor teaching, 
complex clinical examination, limited patient exposure, difficult neuroanatomy, and diagnostic complexity as the major factors in the development of neurophobia(8)(4)(3). These negative perceptions toward neurology can have serious implications, such as decreasing the students' desire to consider neurology as a future career and increasing referral from other specialists to avoid dealing with neurological conditions(9). Fortunately, several methods have been put forward as suggestions for improving teaching of neurology such as : increased tutorials, greater exposure to neurology patients, more neurology lectures, improvements in neuroanatomy teaching, and a mandatory neurology rotations for all medical students(8)(10)(11). Faced with increasing demands of healthcare systems and the universal burden of neurological disorders, there is a rising need for further research and innovative strategies to improve students' perceptions of clinical neurology.

Neurological disorders are considered as one of the major causes of death and disability among affected individuals all over the world. Globally the burden of neurological diseases has increased remarkably in the past few decades (12). According to the world health organization (WHO), neurological diseases represent about $6.3 \%$ of global morbidity and contribute to about $12 \%$ of global mortality(13). A high percentage of these disorders are chronic conditions and usually seen and dealt with by general practitioners in outpatient clinics. Therefore, it is crucial that medical students' as well as medical doctors become more familiar and confident when dealing with those patients. Neurophobia or the unfavorable attitude toward neurosciences and clinical neurology, is prevailing and widely observed among medical students and junior doctors. Neurology is considered, worldwide, as the most difficult and least comprehensible medical specialty. Medical students and general practitioners show less mastery and limited confidence when dealing with neurological cases, while this is not the trend in addressing other medical conditions. In the previous different multinational studies several factors have been implicated in developing this negative attitude toward neurology, and in turn many strategies have been suggested to improve the students' perception of neurology.

In Sudan, little is known about the attitude of medical students toward neurology and the magnitude of neurophobia in the medical community. This study aims to explore this phenomenon, its extent among medical students and most importantly to provide suggestions for improving the attitude of medical students towards this demanding specialty.

\section{Methods}

This was descriptive facility based Cross-sectional study, conducted among medical students at Faculty of Medicine, University of Khartoum, Khartoum state, Sudan 2018.

Medical students of the third, fourth, fifth, sixth years were included in this study. On the other hand, students of the first and the second years were excluded as they have not yet been taught the course of neurosciences. The sample was selected using stratified, systematic random sampling technique (each academic year is considered as a different stratum in the entire population of the students). The sample size was 310. The sample size constituted $22.1 \%$ (310) of the total population (1400). Interval of 4 . 
Data was collected using validated questionnaire which was adopted from previous studies $(2,5)$ consist of four major domains in addition to socio-demographic data (gender and academic year) was designed. The first part of the questionnaire investigated the students' interest in Neurology as a future career using a scale from 1 to $5(1=$ little or no interest, $2=$ some interest, $3=$ moderate interest, 4 = quite interested, $5=$ strongly interested). The second part enquired about the students' satisfaction with the neurosciences course delivered during the first half of the third year. A scale from 1 to 5 was used to evaluate satisfaction ( 1 = strongly unsatisfied, 2 = unsatisfied, $3=$ neutral, 4 = satisfied, 5 = strongly satisfied).

In the third section of the questionnaire students were asked to rate several factors - compiled from many previous studies- according to their effect on the attitude towards neurology. These factors - as listed in the questionnaire - are: 1-Neurology is difficult 2-There isn't enough teaching 3-It is badly taught 4-The difficulty is attributed to the need to know basic neurosciences 5-Insufficient knowledge of the students about Neurology 6-Separation of basic sciences teaching and clinical application 7-There is limited exposure to neurological patients 8- Complex clinical examination 9-Large number of diagnoses 10Difficulty of neuroanatomy 11-Limited treatments 12-Poor prognosis 13-Neurological disorders more difficult emotionally than neurological diseases 14-Very long training time 15-Reputation as a difficult subject 16-Others (i.e., other factors to be mentioned by the respondents). A scale from 1 to 5 was applied ( 1 = strongly disagree, 2 = disagree, 3 = neutral, 4 = agree, 5 = strongly agree).

The fourth part of the questionnaire suggest several strategies to improve Neurology teaching and ask the students to rate them according to their usefulness using a scale from 1 to 5 ( 1 = least useful, $5=$ extremely useful). The investigated strategies are: 1 -Increase the number of bedside tutorials 2 -Increase exposure to neurological patients 3-Increase the lectures 4-Improving teaching skills of the lecturers 5Mandatory neurology rotation for all medical students 6-More time devoted to the teaching of the subject 7-Small group sessions 8-Improvement in neuroanatomy teaching specifically 9-Three dimensional simulators, video recordings and online resources 10-0thers (i.e.: a free margin for other suggestions by the respondents).

Ethical approval was obtained from department of Community Medicine, Faculty of Medicine, University of Khartoum. informed Consent was obtained from all participants prior to data collection, codes were used instead of names to insure confidentiality.

Data was analyzed using SPSS-23 (Statistical Package for Social Sciences version 23). Categorical variables were presented as frequencies and percentages and continuous variables as mean and standard deviation. ttest was used to test the differences between means. P.value less than .05 was considered to be significant.

All methods were carried out in accordance with relevant guidelines and regulations

\section{Results}


A total of 310 participants were finally analyzed. Female constituted $71 \%$ of the participants. 80 (25.8\%) of them were a third year medical students. Table 1

Table 1

Socio-demographic characteristics

\begin{tabular}{|ll|}
\hline \multicolumn{3}{|l|}{} & Frequency (\%) \\
\hline Gender & \\
\hline Male & $90(29 \%)$ \\
\hline Female & $220(71 \%)$ \\
\hline Academic year \\
\hline Third year & $80(25.8 \%)$ \\
\hline Fourth year & $73(23.5 \%)$ \\
\hline Fifth year & $78(25.2 \%)$ \\
\hline Sixth year & $79(25.5 \%)$ \\
\hline
\end{tabular}

The level of interest varied between the students with mean score of 2.37 (+/- 1.35). 37.4\% of the students had little or no interest and only $9.4 \%$ were strongly interested in Neurology. There was no significant difference between male and females in their interest $(p=0.534)$, however a significant statistical association was detected between the level of interest and academic year $(p=0.021)$. It is clear that the level of interest decline as students get onto the advanced years of the college. The second part of the questionnaire enquired about the students' satisfaction with the neurosciences course delivered during the first half of the third year. A scale from 1 to 5 was used to evaluate satisfaction $(1=$ strongly unsatisfied, $5=$ strongly satisfied). The mean score was $2.89(+/-0.918) .46 .8 \%$ of the students graded their satisfaction with the course as neutral (i.e.: moderate) and $30.6 \%$ were unsatisfied with the delivered course $(6.8 \%$ of them were strongly unsatisfied). The statistical association between satisfaction and future interest in Neurology was explored however it was not found significant $(p=0.185)$.

Students were asked to rate several factors - compiled from many previous studies- according to their effect on the attitude towards neurology. A scale from 1 to 5 was applied ( $1=$ strongly disagree, $5=$ strongly agree). The mean scores for the different influencing factors are listed in Table 2 
Table 2

The influencing factors on the attitude towards Neurology

\begin{tabular}{|l|cc|}
\hline & Mean & Standard \\
\hline There is limited exposure to neurological patients & 3.64 & Deviation \\
\hline Separation of basic sciences teaching and clinical application & 3.52 & 1.114 \\
\hline $\begin{array}{l}\text { Neurological disorders more difficult emotionally than non-neurological } \\
\text { diseases }\end{array}$ & 3.45 & 1.113 \\
\hline Insufficient knowledge of the students about Neurology & 3.45 & .932 \\
\hline The difficulty is attributed to the need to know basic neurosciences & 3.43 & .962 \\
\hline Reputation as a difficult subject & 3.36 & 1.014 \\
\hline Poor prognosis & 3.29 & 1.052 \\
\hline Very long training time & 3.28 & 1.156 \\
\hline Neurology is difficult & 3.26 & 1.024 \\
\hline There isn't enough teaching & 3.25 & 1.063 \\
\hline Large number of diagnoses & 3.08 & 1.085 \\
\hline Limited treatments & 3.08 & 1.015 \\
\hline Difficulty of neuroanatomy & 3.06 & 1.181 \\
\hline Complex clinical examination & 2.96 & 1.086 \\
\hline It is badly taught & 2.85 & 1.056 \\
\hline
\end{tabular}

Limited exposure to neurological patients and separation of basic sciences teaching from clinical application were at the top of the list. On the other hand, bad teaching and complex clinical examination were rated as the least influencing factors. Responses in the open-ended question focused on the short period -a single semester- dedicated to deliver a neuroscience course of tremendous amount of information as an influential factor on the attitude of medical students towards Neurology in Khartoum University. An interesting finding was the existence of significant statistical association between academic year and considering the difficulty of neuroanatomy as a remarkable influencing factor on the attitude towards Neurology $(p=0.004)$. Neuroanatomy is considered more difficult as the student proceed into the advanced years of the medical school and hence leads to unfavorable attitude towards Neurology.

The fourth part of the questionnaire suggest several strategies to improve Neurology teaching and ask the students to rate them according to their usefulness using a scale from 1 to 5 ( 1 = least useful, $5=$ extremely useful). The mean scores for the suggested strategies are listed in Table 3 
Table 3

Strategies suggested to improve Neurology teaching

\begin{tabular}{|lll|}
\hline & Mean & \multicolumn{1}{c|}{$\begin{array}{l}\text { Standard } \\
\text { Deviation }\end{array}$} \\
\hline Increase exposure to neurological patients & 4.31 & .935 \\
\hline Three dimensional simulators, video recordings and online resources & 4.24 & 1.018 \\
\hline Improving teaching skills of the lecturers & 4.23 & 1.101 \\
\hline Increase the number of bedside tutorials & 3.94 & 1.171 \\
\hline Small group sessions & 3.89 & 1.256 \\
\hline Mandatory neurology rotation for all medical students & 3.83 & 1.222 \\
\hline Improvement in neuroanatomy teaching specifically & 3.67 & 1.260 \\
\hline More time devoted to the teaching of the subject & 3.54 & 1.192 \\
\hline Increase the lectures & 2.75 & 1.231 \\
\hline
\end{tabular}

Increase exposure to neurological patients (53.2\%), three dimensional simulators, video recordings and online resources $(54.2 \%)$ and improving teaching skills of the lecturers $(58.4 \%)$ were considered as the most helpful and fruitful strategies to improve Neurology teaching. Increasing the lectures was seen as the least useful strategy (10.3). Improvements in neuroanatomy teaching specifically was frequently considered to be much more helpful in the advanced years students when compared to their junior counterparts. A finding that is well supported by the probably underpinning perception of increasing difficulties in neuroanatomy as the students advance forward.

The suggestions of the student in the open-ended question include provision of more developed teaching methods to improve integration between neurosciences and clinical practice, decreasing the number of students in each clinical round group, teaching using problem solving methods, improvements in neuroradiology and neuropharmacology teaching.

\section{Discussion}

This cross-sectional study was designed to explore the attitude of medical students toward Neurology in Khartoum University and to address the issue of Neurophobia which is already established as a concerning phenomenon among different medical schools worldwide. Several multinational studies were conducted to investigate students' attitude toward neurology teaching and practice focusing on risk factors and possible solutions. (2)(3)(4)(5)(6)(7). The fear of neurosciences and clinical Neurology is accused of having serious repercussions and negative impacts on students' future career choices as it discourages medical students from pursuing the demanding Neurology specialties which really suffer from the lack of trained physicians. In this study only $9.4 \%$ were strongly interested in Neurology as a 
future career moreover advanced years students were less likely to choose Neurology specialty in the future $(p=0.021)$ and these results are consistent with the results of a similar study conducted among Saudi medical students ( $11.8 \%$ of Saudi students would consider Neurology as their future specialty and similarly advanced clinical year students -namely interns- were more likely not to consider Neurology as a career choice $(p=0.001))$. (2) However, there was a significant difference between Saudi males and females in their interest in Neurology $(p=0.002)$, a finding that was not detected in our study $(p=0.534)$.

In the attempt to discover the reasons of this undesirable attitude towards Neurology among medical students in Khartoum University, we asked the participants to rate several factors - derived from previous studies - known to contribute to development of the Neurophobia phenomenon. The students in our study selected limited exposure to neurological patients and separation of basic sciences teaching from clinical application as the most influencing factors. These results are consistent with other studies carried out in United Kingdom, Ireland, United states as well as the Caribbean region (10) (4)(5)(3). Surprisingly our students downplayed the effect of bad teaching and complex clinical examination on the attitude towards Neurology, these finding oddly contradict the conclusions of the previously mentioned studies conducted internationally in different parts of the world and even regionally conducted survey in Saudi Arabia; a neighbor country. A finding which needs further investigations and deeper research in this arena to come up with more clarifications in this regard.

A point worth to mention is the known relationship between neuroanatomy and Neurophobia among medical students as it considered a major contributor to why students perceive neurology as a difficult field. This relationship was reported in various studies implemented in several medical schools all over the world (2)(3)(4)(5) (10). In our study this association between Neuroanatomy and students' attitude towards Neurology is exist $(p=0.004)$ and this finding resembles what is found in other studies. However, neuroanatomy was considered more difficult by advanced years students, and this finding clearly opposes the results of American study in which neuroanatomy was considered less difficult among residents when compared to students. (5)

Eventually our study tried to shed light on how to improve Neurology teaching. Increase exposure to neurological patients, three dimensional simulators, video recordings, online resources and improving teaching skills of the lecturers was considered as the most useful strategies. These results reinforce the outcomes of a review discussed evidence-based recommendations and educational interventions to cure neurophobia in medical education, One of these interventions was the use of three-dimensional simulators, video recordings, and online resources in teaching neurological examination (9). In a similar study conducted in US the participants reported that online resources, textbooks and bedside teaching were useful or extremely useful methods when learning Neurology (5). In a British study four main answers were given regarding the question of how to improve Neurology teaching. The commonest was the need for more teaching, with half of the participants specifying the need for more clinical teaching. Next came the need to integrate basic neuroscience teaching with clinical neurology. Third was the need for better teaching, with half the respondents specifically mentioning neuroscience teaching. Fourth was the need to make clear what is "simple, basic, straightforward, and important" (10). In China students 
rated bedside teaching as having the greatest value in learning neurology, this was followed by smallgroup teaching (7). An Irish study determined increase tutorials, greater exposure to neurological patients, more lectures and mandatory neurology rotations as the most helpful methods. video teaching and online resources usefulness is underrated among Irish students (7). In a study carried out in the Caribbean region, students' responses clustered around 4 areas: building more clinical or practical exposure into the teaching, more time was necessary to be devoted to the teaching of the subject, need for improved teaching tools, in particular audio-visual equipment as well as the need for the actual lecturing skills of the teachers to be improved or teaching needing to be better organized (3). Increasing the lectures was seen as the least useful strategy in our study, a similar result was also detected in the American study (5). Improvements in neuroanatomy teaching was recommended in most of the previously mentioned studies as well as our own study however the demand for improvement was more obvious among advanced years' students in comparison to their junior counterparts, a finding that match the increased perception of the difficulty of neuroanatomy among the senior students.

\section{Conclusion}

A cross sectional study was conducted in the Faculty of Medicine, University of Khartoum in 2018 aiming to study the attitude of medical students towards Neurology and to explore the phenomenon of neurophobia, the results of it can be recapitulated in the following points:

Students had unfavorable interest towards Neurology and expectedly a modest proportion of the students would pursue Neurology specialty in the future.

Students' satisfaction with the neurosciences courses was rated as moderate to poor in the majority of the responses.

Limited exposure to neurological patients and separation of basic sciences teaching from clinical application were considered as the top contributing factors to creation of this negative perception towards Neurology.

Students' opinions regarding the most efficient ways to improve Neurology teaching have cited increased exposure to neurological patients, three dimensional simulators, video recordings, online resources and improving teaching skills of the lecturers as the most helpful and fruitful strategies to make progress in this arena.

\section{Recommendations}

1. Conducting more researches in this topic by the education development centers or the concerned stakeholders in the different institutions all over Sudan with the aim to reach more solid and generalizable results.

2. Innovation of new strategies to encourage the medical students to pursue the demanding field of Neurology which ails from lack of training doctors. 
3. Leading more deep endeavors to fathom the reasons beyond the unfavorable attitude towards Neurology and trying to tackle them using evidence -based interventions.

4. Implementation of three-dimensional simulators, video recordings, online resources, improved teaching skills of the lecturers and increase exposure to neurological patients to make a potential breakthrough in Neurology teaching.

\section{Abbreviations}

WHO World Health Organization

SPSS Statistical Package of Social Sciences

\section{Declarations}

Ethical approval and consent to participate

The study was approved by Local ethical committee at the Department of Community Medicine, University of Khartoum. Informed consent was obtained from all participants before data collection.

Consent for Publication

Not applicable

Availability of data and materials

The data-sets used during the current study are available in a supplementary file.

Competing Interests

The authors declare that they have no competing interests.

\section{Funding}

No Fund

Author's contributions

AA provide the concept of the study and was the major contributor in writing the manuscript. MM analyzed and interpret respondent's data. All authors read and approved the final manuscript.

Acknowledgment:

We would like to express our deep sense of gratitude to Dr. Sayed Halali, Dr. Khalid Abdelsalam,Basil Abubakr, Siham Abdulrahman, Yusra Alsayed, Zeinab Elmamoun ,Areej Alsayed, Areej Mutasim, 
Mohammed Abdullah, Ahmed Daffa-Allah Musaad -and Abdulmalik Ameen for their endless help and suport.

\section{References}

1. Jozefowicz RF. Neurophobia : the fear of neurology among medical students. Arch Neurol. 1994;51:328-329

2. Abulaban AA, Obeid TH, Algahtani HA, Kojan SM. Neurophobia among medical students. 2015;20:37-40.

3. Youssef FF. Neurophobia and its implications: evidence from a Caribbean medical school. 2009;7:17.

4. Flanagan E, Walsh C, Tubridy N. Ô Neurophobia Õ - attitudes of medical students and doctors in Ireland to neurological teaching. 2007;1109-12.

5. Zinchuk A V, Flanagan EP, Tubridy NJ, Miller WA, Mccullough LD. Attitudes of US medical trainees towards neurology education: "Neurophobia " - a global issue. 2010;

6. Albert D V. Correspondence Neurophobia among medical students. 2015;20(4):20150500.

7. Lukas R V, Cooper B, Morgan I, Brorson JR. Attitudes Toward Neurosciences in Medical Students in Wuhan, China: A Survey Study. World Neurosurg [Internet]. Elsevier Ltd; 2014; Available from: http://dx.doi.org/10.1016/j.wneu.2014.06.025

8. Lim ECH, Seet RCS. Demystifying neurology: preventing " neurophobia " among medical students. 2008;4(8):462-3.

9. .Abdelrahman Ibrahim Abushouk \& Nguyen Minh Duc (2016) Curing neurophobia in medical schools: evidence-basedstrategies, Medical Education Online, 21:1, 32476, DOI: 10.3402/meo.v21.32476

10. Schon F, Hart P, Fernandez C. Is clinical neurology really so difficult? :557-9.

11. Shiels L, Majmundar P, Zywot A, Sobotka J, Lau CSM, Jalonen TO. Medical student attitudes and educational interventions to prevent neurophobia: a longitudinal study. BMC Medical Education; 2017;1-7.

12. Bill F, Foundation MG. Articles Global , regional , and national burden of neurological disorders during 1990 - 2015: a systematic analysis for the Global Burden of Disease Study 2015. 2017;

13. Daly O, Gbd T. Neurological disorders: public health challenges Projections of global mortality and burden of disease.

\section{Supplementary Files}

This is a list of supplementary files associated with this preprint. Click to download.

- neurophobia.sav 